POS $\quad$ PROCEEDINGS

\title{
Towards a determination of the ratio of the kaon to pion decay constants
}

\author{
V. G. Bornyakov ${ }^{a}$, R. Horsley ${ }^{* b}$, Y. Nakamura ${ }^{c}$, H. Perlt ${ }^{d}$, D. Pleiter ${ }^{e}$, P. E. L. Rakow ${ }^{f}$, \\ G. Schierholz ${ }^{g}$, A. Schiller ${ }^{d}$, H. Stüben ${ }^{h}$ and J. M. Zanotti ${ }^{i}$ \\ ${ }^{a}$ Institute for High Energy Physics, Protvino, 142281 Protvino, Russia, \\ Institute of Theoretical and Experimental Physics, Moscow, 117259 Moscow, Russia, \\ School of Biomedicine, Far Eastern Federal University, 690950 Vladivostok, Russia \\ ${ }^{b}$ School of Physics and Astronomy, University of Edinburgh, Edinburgh EH9 3FD, UK \\ ${ }^{c}$ RIKEN Advanced Institute for Computational Science, Kobe, Hyogo 650-0047, Japan \\ ${ }^{d}$ Institut für Theoretische Physik, Universität Leipzig, 04109 Leipzig, Germany \\ e JSC, Forschungszentrum Jülich, 52425 Jülich, Germany, \\ Institut für Theoretische Physik, Universität Regensburg, 93040 Regensburg, Germany \\ ${ }^{f}$ Theoretical Physics Division, Department of Mathematical Sciences, University of Liverpool, \\ Liverpool L69 3BX, UK \\ ${ }^{g}$ Deutsches Elektronen-Synchrotron DESY, 22603 Hamburg, Germany \\ ${ }^{h}$ Universität Hamburg, Regionales Rechenzentrum, 20146 Hamburg, Germany \\ ${ }^{i}$ CSSM, Department of Physics, University of Adelaide, Adelaide SA 5005, Australia \\ E-mail: rhorsley@ph.ed.ac.uk
}

\section{QCDSF-UKQCD Collaborations}

The SU(3) flavour symmetry breaking expansion in up, down and strange quark masses is extended from hadron masses to meson decay constants. This allows a determination of the ratio of kaon to pion decay constants in QCD. Furthermore when using partially quenched valence quarks the expansion is such that $\mathrm{SU}(2)$ isospin breaking effects can also be determined. It is found that the lowest order SU(3) flavour symmetry breaking expansion (or Gell-Mann-Okubo expansion) works very well. Simulations are performed for $2+1$ flavours of clover fermions at four lattice spacings.

34th annual International Symposium on Lattice Field Theory

24-30 July 2016

University of Southampton, UK

\footnotetext{
* Speaker.
} 


\section{Introduction/Approach}

The QCD interaction is flavour-blind. Neglecting electromagnetic and weak interactions, the only difference between flavours comes from the mass matrix. In this talk we want to look at how this constrains meson decay matrix elements once full $S U$ (3) flavour symmetry is broken, using the same methods as we used in $[1,2]$ for hadron masses. In particular we shall consider pseudoscalar decay matrix elements and give an estimation for $f_{K} / f_{\pi}$ (and $f_{K^{+}} / f_{\pi^{+}}$ignoring electromagnetic contributions).

In lattice simulations with three dynamical quarks there are many paths to approach the physical point where the quarks take their physical values. The choice adopted here is to extrapolate from a point on the $S U$ (3) flavour symmetry line keeping the the singlet quark mass $\bar{m}$ constant, as illustrated in the left panel of Fig. 1, for the case of two mass degenerate quarks $m_{u}=m_{d} \equiv m_{l}$.
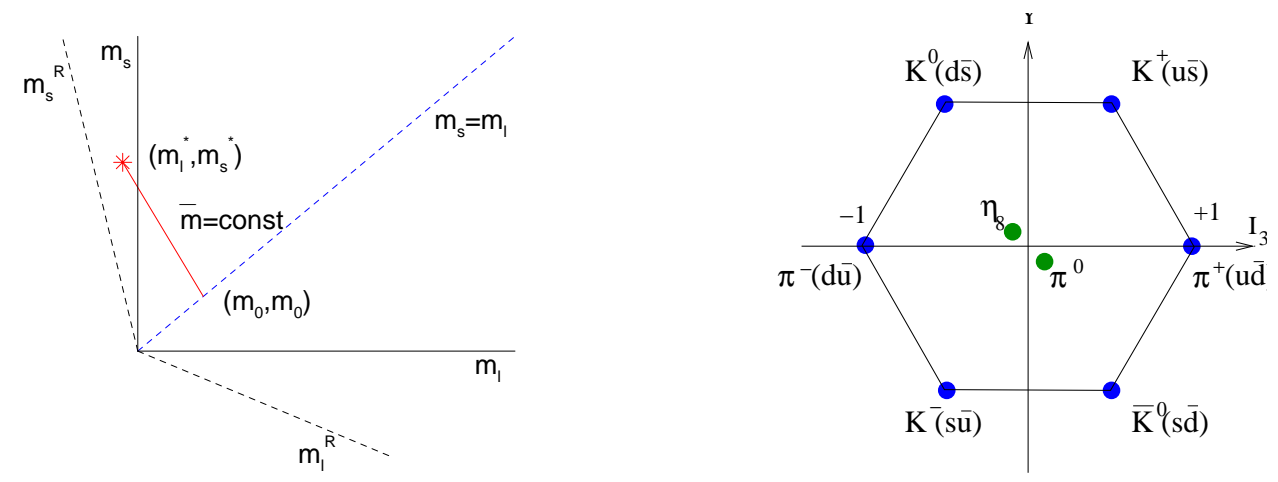

Figure 1: LH panel: Sketch of the path for the case of two mass degenerate quarks, $m_{u}=m_{d} \equiv m_{l}$, from a point on the $S U(3)$ flavour symmetric line $\left(m_{0}, m_{0}\right)$ to the physical point denoted with a * $\left(m_{l}^{*}, m_{s}^{*}\right)$. RH panel: The pseudoscalar octet meson.

This allows the development of an $S U(3)$ flavour symmetry breaking expansion for hadron masses and matrix elements, i.e. an expansion in

$$
\delta m_{q}=m_{q}-\bar{m}, \quad \text { with } \quad \bar{m}=\frac{1}{3}\left(m_{u}+m_{d}+m_{s}\right),
$$

(where numerically $\bar{m}=m_{0}$ ). From this definition we have the trivial constraint $\delta m_{u}+\delta m_{d}+$ $\delta m_{s}=0$. The path to the physical quark masses is called the 'unitary line' as we expand in the same masses for the sea and valence quarks. Note also that the expansion coefficients are functions of $\bar{m}$ only, which provided we keep $\bar{m}=$ const. reduces the number of allowed expansion coefficients considerably.

As an example of an $S U(3)$ flavour symmetry breaking expansion, [2], we consider the pseudoscalar masses and find to NLO (i.e. $O\left(\left(\delta m_{q}\right)^{2}\right)$ )

$$
\begin{aligned}
M^{2}(a \bar{b})=M_{0}^{2}+ & \alpha\left(\delta m_{a}+\delta m_{b}\right) \\
& +\beta_{0} \frac{1}{6}\left(\delta m_{u}^{2}+\delta m_{d}^{2}+\delta m_{s}^{2}\right)+\beta_{1}\left(\delta m_{a}^{2}+\delta m_{b}^{2}\right)+\beta_{2}\left(\delta m_{a}-\delta m_{b}\right)^{2}+\ldots
\end{aligned}
$$

where $m_{a}, m_{b}$ are quark masses with $a, b=u, d, s$. This describes the physical outer ring of the pseudoscalar meson octet (the right panel of Fig. 1). Numerically we can also in addition consider 
a fictitious particle, where $a=b=s$, which we call $\eta_{s}$. We have further determined the expansion to NNLO. (Octet baryons also have equivalent expansions.) The vacuum is a singlet, so meson to vacuum matrix elements $\langle 0|\widehat{\mathscr{O}}| M\rangle$ are proportional to $1 \otimes 8 \otimes 8$ tensors, i.e. $8 \otimes 8$ matrices, where $\widehat{\mathscr{O}}$ is an octet operator. So the allowed mass dependence of the outer ring octet decay constants is similar to the allowed dependence of the octet masses. Thus we have

$$
\begin{aligned}
f(a \bar{b})=F_{0}+ & G\left(\delta m_{a}+\delta m_{b}\right) \\
& +H_{0} \frac{1}{6}\left(\delta m_{u}^{2}+\delta m_{d}^{2}+\delta m_{s}^{2}\right)+H_{1}\left(\delta m_{a}^{2}+\delta m_{b}^{2}\right)+H_{2}\left(\delta m_{a}-\delta m_{b}\right)^{2}+\ldots
\end{aligned}
$$

The $S U(3)$ flavour symmetric breaking expansion has the simple property that for any flavour singlet quantity, which we generically denote by $X_{S} \equiv X_{S}\left(m_{u}, m_{d}, m_{s}\right)$ then

$$
X_{S}\left(\bar{m}+\delta m_{u}, \bar{m}+\delta m_{d}, \bar{m}+\delta m_{s}\right)=X_{S}(\bar{m}, \bar{m}, \bar{m})+O\left(\left(\delta m_{q}\right)^{2}\right) .
$$

This is already encoded in the above pseudoscalar $S U(3)$ flavour symmetric breaking expansions, or more generally it can be shown that $X_{S}$ has a stationary point about the $S U(3)$ flavour symmetric line. Here we shall consider

$$
\begin{aligned}
X_{\pi}^{2} & =\frac{1}{6}\left(M_{K^{+}}^{2}+M_{K^{0}}^{2}+M_{\pi^{+}}^{2}+M_{\pi^{-}}^{2}+M_{\bar{K}^{0}}^{2}+M_{K^{-}}^{2}\right), \\
X_{f_{\pi}} & =\frac{1}{6}\left(f_{K^{+}}+f_{K^{0}}+f_{\pi^{+}}+f_{\pi^{-}}+f_{\bar{K}^{0}}+f_{K^{-}}\right) .
\end{aligned}
$$

(The experimental value of $X_{\pi}$ is $\sim 410 \mathrm{MeV}$, which sets the 'extrapolation' range.) There are, of course, many other possibilities such as $S=N, \Lambda, \Sigma^{*}, \Delta, \rho, r_{0}, t_{0}, w_{0},[1,2,3]$. As a further check, it can be shown that this property also holds using chiral perturbation theory. For example for mass degenerate $u$ and $d$ quark masses and assuming $\chi \mathrm{PT}$ is valid in the region of the $S U(3)$ flavour symmetric quark mass we find

$$
X_{f_{\pi}}=f_{0}\left[1+\frac{8}{f_{0}^{2}}\left(3 L_{4}+L_{5}\right) \bar{\chi}-3 L(\bar{\chi})\right]+O\left(\left(\delta \chi_{l}\right)^{2}\right)
$$

where the expansion parameter is given by $\delta \chi_{l}=\bar{\chi}-\chi_{l}$ with $\bar{\chi}=\frac{1}{3}\left(2 \chi_{l}+\chi_{s}\right), \chi_{l}=B_{0} m_{l}, \chi_{s}=$ $B_{0} m_{s}$, where $f_{0}$ is the pion decay constant in the chiral limit, $L_{i}$ are chiral constants and $L(\chi)=$ $\chi /\left(4 \pi f_{0}\right)^{2} \times \ln \left(\chi / \Lambda_{\chi}^{2}\right)$ is the chiral logarithm.

The unitary range is rather small so we introduce PQ or partially quenching (i.e. the valence quark masses can be different to the sea quark masses), without increasing the number of expansion coefficients. Let us denote the valence quark masses by $\mu_{q}$ and the expansion parameter as $\delta \mu_{q}=$ $\mu_{q}-\bar{m}$. Then we find

$$
\begin{aligned}
\tilde{M}^{2}(a \bar{b})= & 1+\tilde{\alpha}\left(\delta \mu_{a}+\delta \mu_{b}\right) \\
& -\left(\frac{2}{3} \tilde{\beta}_{1}+\tilde{\beta}_{2}\right)\left(\delta m_{u}^{2}+\delta m_{d}^{2}+\delta m_{s}^{2}\right)+\tilde{\beta}_{1}\left(\delta \mu_{a}^{2}+\delta \mu_{b}^{2}\right)+\tilde{\beta}_{2}\left(\delta \mu_{a}-\delta \mu_{b}\right)^{2}+\ldots,
\end{aligned}
$$

and

$$
\begin{aligned}
\tilde{f}(a \bar{b})= & 1+\tilde{G}\left(\delta \mu_{a}+\delta \mu_{b}\right) \\
& -\left(\frac{2}{3} \tilde{H}_{1}+\tilde{H}_{2}\right)\left(\delta m_{u}^{2}+\delta m_{d}^{2}+\delta m_{s}^{2}\right)+\tilde{H}_{1}\left(\delta \mu_{a}^{2}+\delta \mu_{b}^{2}\right)+\tilde{H}_{2}\left(\delta \mu_{a}-\delta \mu_{b}\right)^{2}+\ldots,
\end{aligned}
$$

where in addition to the PQ generalisation we have also formed the ratios $\tilde{M}^{2}=M^{2} / X_{\pi}^{2}, \tilde{\alpha}=\alpha / M_{0}^{2}$, $\ldots$ and $\tilde{f}=f / X_{f_{\pi}}, \tilde{G}=G / F_{0}, \ldots$ This will later prove useful for the numerical results. We see that there are mixed sea/valence mass terms at NLO (and higher orders). The unitary limit is recovered by simply replacing $\delta \mu_{q} \rightarrow \delta m_{q}$. 


\section{The Lattice}

We use an $O(a)$ NP improved clover action with tree level Symanzik glue and mildly stout smeared $2+1$ clover fermions, [4], for $\beta \equiv 10 / g_{0}^{2}=5.40,5.50,5.65,5.80$ (four spacings). We set

$$
\mu_{q}=\frac{1}{2}\left(\frac{1}{\kappa_{q}^{\mathrm{val}}}-\frac{1}{\kappa_{0 c}}\right), \quad \text { giving } \delta \mu_{q}=\mu_{q}-\bar{m}=\frac{1}{2}\left(\frac{1}{\kappa_{q}^{\mathrm{val}}}-\frac{1}{\kappa_{0}}\right) .
$$

A $\kappa$ value along the $S U(3)$ symmetric line is denoted by $\kappa_{0}$, while $\kappa_{0 c}$ is the value in the chiral limit. Note that practically we do not have to determine $\kappa_{0 c}$, as it cancels in $\delta \mu_{q}$. (For simplicity we have set the lattice spacing to unity.)

We first investigate the constancy of $X_{S}$ in the unitary region. In the left hand panel of Fig 2 we show various $X_{S}$ s. It is apparent that over a large range, starting from the $S U(3)$ flavour symmetric
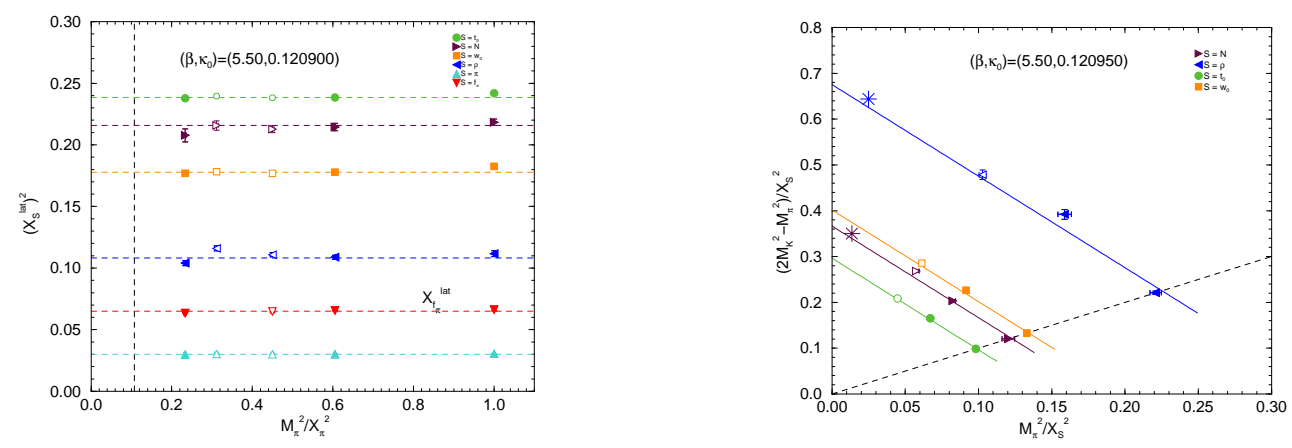

Figure 2: LH panel: $X_{t_{0}}^{2}, X_{w_{0}}^{2}, X_{\pi}^{2}, X_{\rho}^{2}, X_{N}^{2} \approx X_{\Lambda}^{2}, X_{f_{\pi}}$ for $\left(\beta, \kappa_{0}\right)=(5.50,0.120900)$ along the $\bar{m}=$ const. line, together with constant fits. Open symbols have $M_{\pi} L \lesssim 4$ and are not included in the fit. The vertical line is the physical point. RH panel: $\left(2 M_{K}^{2}-M_{\pi}^{2}\right) / X_{S}^{2}$ versus $M_{\pi}^{2} / X_{S}^{2}, S=N, \rho, t_{0}, w_{0}$ for $\left(\beta, \kappa_{0}\right)=(5.50,0.120950)$. Stars represent the physical points, the dashed line is the $S U(3)$ flavour symmetric line.

line, reaching down and approaching the physical point, $X_{S}$ appears constant, with very little evidence of curvature. Based on this observation, we determine the path in the quark mass plane by considering $M_{\pi}^{2} / X_{S}^{2}$ against $\left(2 M_{K}^{2}-M_{\pi}^{2}\right) / X_{S}^{2}$. If there is little curvature then we expect that

$$
\frac{2 M_{K}^{2}-M_{\pi}^{2}}{X_{S}^{2}}=3 \frac{X_{\pi}^{2}}{X_{S}^{2}}-2 \frac{M_{\pi}^{2}}{X_{S}^{2}}
$$

holds for $S=N, \rho, t_{0}, w_{0}, \ldots$ In the right panel of Fig 2 we show this for $\left(\beta, \kappa_{0}\right)=(5.50,0.120950)$. We see that this is indeed the case. $\kappa_{0}$ is adjusted so that the path goes through the physical value. (For example, $\beta=5.50, \kappa_{0}=0.120950$ is much closer to this path than $\kappa_{0}=0.120900$, see [3].)

The programme is thus first to determine $\kappa_{0}$ and then find the expansion coefficients. Then use $^{1}$ isospin symmetric "physical' masses $M_{\pi}^{* 2}, M_{K}^{* 2}$ to determine $\delta m_{l}^{*}$ and $\delta m_{s}^{*}$. PQ results can help for the first task. As the range of PQ quark masses that can then be used is much larger than the unitary range, then the numerical determination of the expansion coefficients is improved. In Fig. 3 we show $\tilde{M}_{\pi}^{2}$ and $\tilde{f}$ against $\delta \mu_{a}+\delta \mu_{b}$. From previous results the LO expansions are just a

\footnotetext{
${ }^{1}$ Masses are taken from FLAG3, [5].
} 

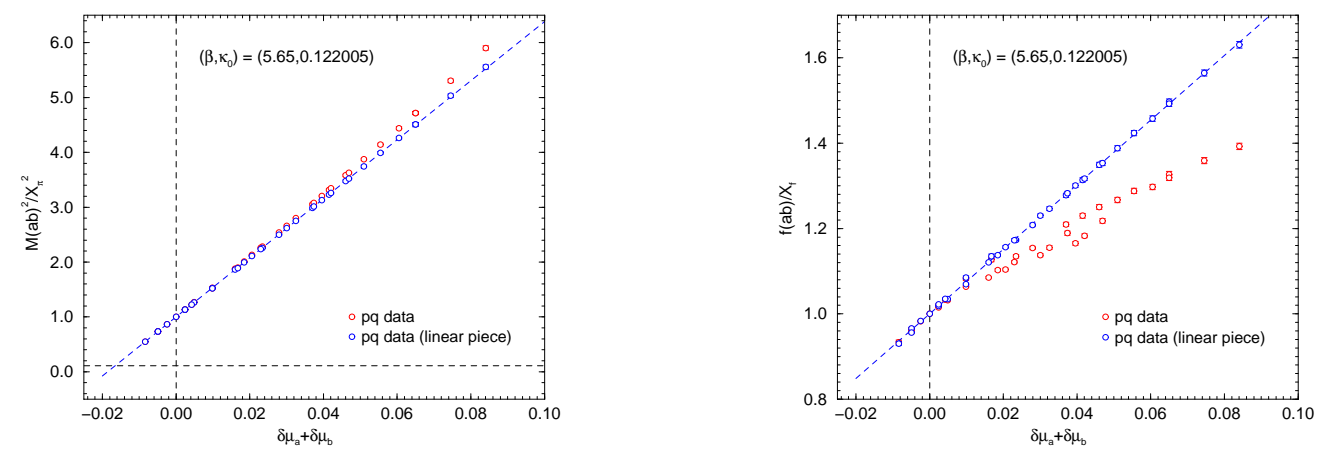

Figure 3: LH panel: PQ (and unitary) pseudoscalar mass results for $\tilde{M}^{2}=M^{2} / X_{\pi}^{2}$ with $\left(\beta, \kappa_{0}\right)=$ $(5.65,0.122005)$ against valence quarks $\delta \mu_{a}+\delta \mu_{b}$. The data is given by red circles, while subtracting out the non-linear pieces (using the fit) gives the blue circles, together with the linear fit. The vertical dashed line is the symmetric point, while the horizontal dashed line represents the physical $\tilde{M}_{\pi}^{* 2}$. RH panel: Similarly for the decay constant, $\tilde{f}=f / X_{f_{\pi}}$.

function of $\delta \mu_{a}+\delta \mu_{b}$; at higher orders, NLO etc., this is not the case. We see that there is linear behaviour in the masses at least for $\tilde{M}_{\pi}^{2} \lesssim 3$ or $M_{\pi} \lesssim \sqrt{3} \times 410 \mathrm{MeV} \sim 700 \mathrm{MeV}$.

Furthermore the use of PQ results allows for a method for fine tuning of $\kappa_{0}$ to be developed. If we slightly miss the starting point on the $S U(3)$ flavour symmetric line, we can also tune $\kappa_{0}$ using PQ results so that we get the physical values of (say) $M_{\pi}^{*}, X_{N}^{*}$ and $M_{K}^{*}$ correct. This gives $\kappa_{0}, \delta \mu_{l}^{*}$, $\delta \mu_{s}^{*}$. The philosophy is that most change is due to a change in valence quark mass, rather than sea quark mass. Note that then $2 \delta \mu_{l}+\delta \mu_{s} \neq 0$ necessarily (while $2 \delta m_{l}+\delta m_{s}$ is always $=0$ ). For our $\kappa_{0}$ values this is a rather small change, which we take to be part of the systematic error. Presently we use $\left(\beta, \kappa_{0}\right)=(5.40,0.119930),(5.50,0.120950),(5.65,0.122005),(5.80,0.122810)$, [3].

\section{Decay constants}

The renormalised and $O(a)$ improved axial current is given by [6]

$$
\mathscr{A}_{\mu}^{a b ; R}=Z_{A} \mathscr{A}_{\mu}^{a b ; \mathrm{IMP}},
$$

with

$$
\mathscr{A}_{\mu}^{a b ; \mathrm{IMP}}=\left(1+\left[\bar{b}_{A} \bar{m}+\frac{1}{2} b_{A}\left(m_{a}+m_{b}\right)\right]\right) \mathscr{A}_{\mu}^{a b}, \quad \mathscr{A}_{\mu}^{a b}=A_{\mu}^{a b}+c_{A} \partial_{\mu} P^{a b},
$$

and

$$
A_{\mu}^{a b}=\bar{q}_{a} \gamma_{\mu} \gamma_{5} q_{b}, \quad P^{a b}=\bar{q}_{a} \gamma_{5} q_{b} .
$$

Using the axial current we first define matrix elements

$$
\left\langle 0\left|\widehat{A}_{4}\right| M\right\rangle=M f, \quad\left\langle 0\left|\widehat{\partial_{4} P}\right| M\right\rangle=M f^{(1)},
$$

giving for the renormalised pseudoscalar constants

$$
f^{R}=Z_{A}\left(1+c_{A} \frac{f^{(1)}}{f}\right)\left(1+\left[\left(\bar{b}_{A}+b_{A}\right) \bar{m}+\frac{1}{2} b_{A}\left(\delta m_{a}+\delta m_{b}\right)\right]\right) f .
$$



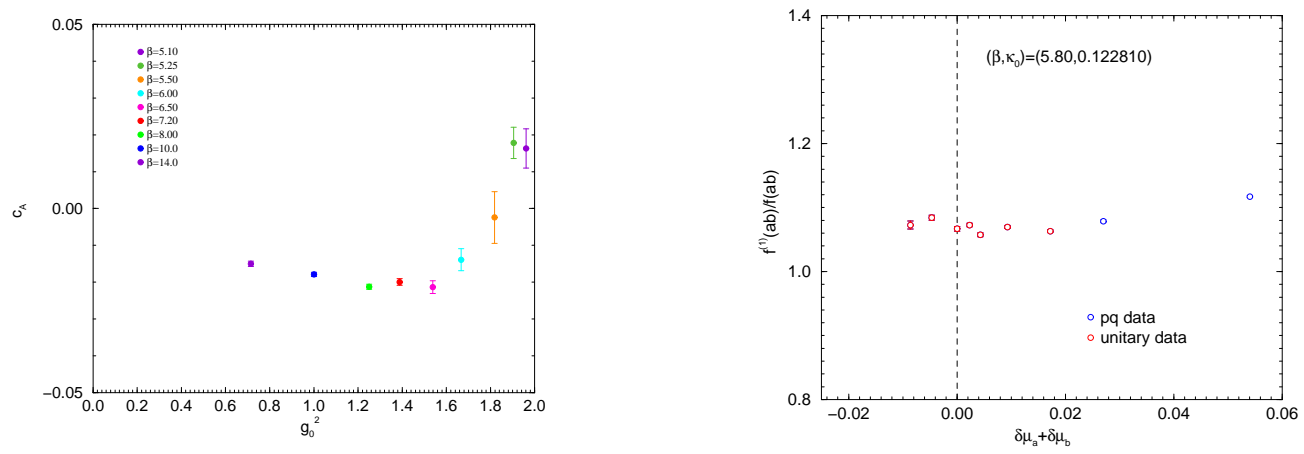

Figure 4: LH panel: Estimate of the $c_{A}$ improvement coefficient using the Schrödinger Functional, [4] as a function of $g_{0}^{2}$. RH panel: The ratio $f^{(1)} / f$ versus $\delta \mu_{a}+\delta \mu_{b}$ for $\left(\beta, \kappa_{0}\right)=(5.80,0.122810)$.

As indicated in Fig. 4, we note that $c_{A}$ is small (compared to unity) and that $f^{(1)} / f$ is constant and $\sim O(1)$. So for constant $\bar{m}$ we can absorb the $c_{A} f^{(1)} / f$ and $\left(\bar{b}_{A}+b_{A}\right) \bar{m}$ terms to give

$$
\tilde{f}^{R} \equiv \frac{f^{R}}{X_{f_{\pi}}^{R}}=1+\left(\tilde{G}+\frac{1}{2} b_{A}\right)\left(\delta m_{a}+\delta m_{b}\right)+\ldots
$$

For $b_{A}$ (only defined up to terms of $O(a)$ ) we presently take the tree level value, $b_{A}=1+O\left(g_{0}^{2}\right)$.

As demonstrated in Fig. 3, we expect LO behaviour to dominate in the unitary region. In the left panel of Fig. 5 we show typical unitary results for $\left(\beta, \kappa_{0}\right)=(5.80,0.122810)$ for $\tilde{f}=f / X_{f_{\pi}}$.
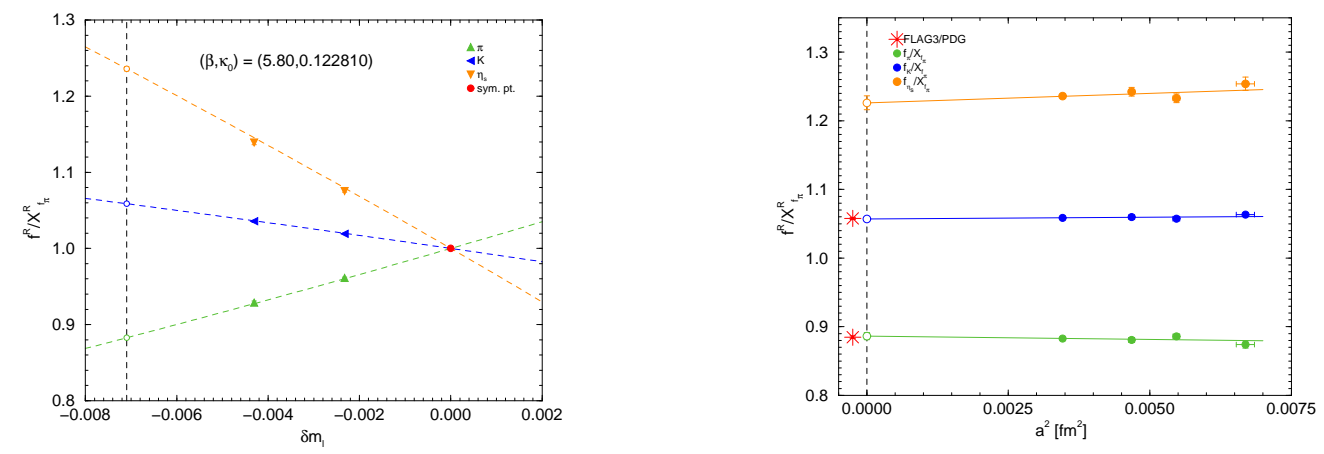

Figure 5: LH panel: Unitary results for $\tilde{f}=f / X_{f_{\pi}}$ versus $\delta m_{l}$ (filled circles) for $\left(\beta, \kappa_{0}\right)=(5.80,0.122810)$. The extrapolated values at the physical quark masses are given as open circles. RH panel: The continuum extrapolation. The extrapolated values are again given as open circles. The converted FLAG3 values, [5], are given as stars.

Finally for our four beta values, we perform the continuum extrapolation, as shown in the right panel of Fig. 5. For comparison, the FLAG3 values, [5], are shown as stars. (Note that although $f_{\eta_{s}}$ helps in determining the expansion coefficients, there is no further information to be found from the various extrapolated values.) Converting $\tilde{f}_{K}$ gives a result of $f_{K} / f_{\pi}=1.192(10)(13)$.

Finally we briefly discuss $S U(2)$ isospin breaking effects. Provided $\bar{m}$ is kept constant, then the $S U(3)$ flavour breaking expansion coefficients $(\tilde{\alpha}, \tilde{G}, \ldots)$ remain unaltered whether we consider $1+1+1$ or $2+1$ flavours. So although our numerical results are for mass degenerate $u$ and $d$ quarks 
we can use them to discuss isospin breaking effects. We parameterise these effects by

$$
\frac{f_{K^{+}}}{f_{\pi^{+}}}=\frac{f_{K}}{f_{\pi}}\left(1+\frac{1}{2} \delta_{S U(2)}\right)
$$

and expanding in $\Delta m=\left(\delta m_{d}-\delta m_{u}\right) / 2$ about the average light quark mass $\delta m_{l}=\left(\delta m_{u}+\delta m_{d}\right) / 2$ gives, using the LO expansions (which from Fig. 3 and the LH panel of Fig. 5 have been shown to work quite well)

$$
\delta_{S U(2)}=\frac{2}{3}\left(1-\left(\frac{f_{K}}{f_{\pi}}\right)^{-1}\right) \frac{\Delta m}{\delta m_{l}}, \quad \text { with } \quad \frac{\Delta m}{\delta m_{l}}=\frac{3}{2} \frac{M_{K^{0}}^{2}-M_{K^{+}}^{2}}{M_{\pi^{+}}^{2}-\frac{1}{2}\left(M_{K^{0}}^{2}+M_{K^{+}}^{2}\right)} .
$$

At the physical point $\Delta m^{*} / \delta m_{l}^{*}=-0.0393$ and hence here $\delta_{S U(2)}=-0.0042(2)(2)$.

\section{Conclusions}

We have extended our programme of tuning the strange and light quark masses to their physical values simultaneously by keeping the average quark mass constant from pseudoscalar meson masses to pseudoscalar decay constants. As for masses we find that the $S U(3)$ flavour symmetry breaking expansion, or Gell-Mann-Okubo expansion, works well even at leading order.

\section{Acknowledgements}

The numerical configuration generation (using the BQCD lattice QCD program [7]) and data analysis (using the Chroma software library [8]) was carried out on the IBM BlueGene/Qs using DIRAC 2 resources (EPCC, Edinburgh, UK), and at NIC (Jülich, Germany), the Lomonosov at MSU (Moscow, Russia) and the SGI ICE 8200 and Cray XC30 at HLRN (The North-German Supercomputer Alliance) and on the NCI National Facility in Canberra, Australia (supported by the Australian Commonwealth Government). HP was supported by DFG Grant No. SCHI 422/10-1. PELR was supported in part by the STFC under contract ST/G00062X/1 and JMZ was supported by the Australian Research Council Grant No. FT100100005 and DP140103067. We thank all funding agencies.

\section{References}

[1] W. Bietenholz et al., [QCDSF-UKQCD Collaboration], Phys. Lett. B690 (2010) 436, [arXiv:1003.1114[hep-lat]].

[2] W. Bietenholz et al., [QCDSF-UKQCD Collaboration], Phys. Rev. D84 (2011) 054509 , [arXiv: $1102.5300[$ hep-lat] ].

[3] V. G. Bornyakov et al., [QCDSF-UKQCD Collaboration], arXiv:1508.05916 [hep-lat].

[4] N. Cundy et al., [QCDSF-UKQCD Collaboration], Phys. Rev. D79 (2009) 094507, [arXiv:0901.3302 [hep-lat]].

[5] S. Aoki et al., arXiv:1607.00299[hep-lat].

[6] T. Bhattacharya et al., Phys. Rev. D73 (2006) 034504, [arXiv: hep-lat/0511014 ].

[7] Y. Nakamura et al., Proc. Sci. Lattice 2010 (2010) 040, arXiv: 1011.0199 [hep-lat ].

[8] R. G. Edwards et al., Nucl. Phys. Proc. Suppl. 140 (2005) 832, arXiv : hep-lat 0409003. 